\title{
MORPHIC: Programmable photonic circuits enabled by silicon photonic MEMS
}

\author{
Wim Bogaerts ${ }^{\mathrm{a}}$, Hamed Sattari ${ }^{\mathrm{b}}$, Pierre Edinger ${ }^{\mathrm{c}}$, Alain Yuji Takabayashi ${ }^{\mathrm{b}}$, Iman Zand ${ }^{\mathrm{a}}$, \\ Xiaojing Wang ${ }^{\mathrm{c}}$, Antonio Ribeiro ${ }^{\mathrm{a}}$, Moises Jezzini ${ }^{\mathrm{d}}$, Carlos Errando-Herranz ${ }^{\mathrm{c}}$, Giuseppe Talli ${ }^{\mathrm{d}}$, \\ Kumar Sauravf, Marco Garcia Porcele ${ }^{\mathrm{e}}$, Peter Verheyen ${ }^{\mathrm{g}}$, Banafsheh Abasahl ${ }^{\mathrm{a}}$, Frank Niklaus ${ }^{\mathrm{c}}$, \\ Niels Quack ${ }^{\mathrm{b}}$, Kristinn B. Gylfason ${ }^{\mathrm{c}}$, Peter O'Brien ${ }^{\mathrm{d}}$, and Umar Khan ${ }^{\mathrm{a}}$ \\ ${ }^{a}$ Ghent University - IMEC, Photonics Research Group, Department of Information \\ Technology,, Technologiepark-Zwijnaarde 126, 9052 Gent, Belgium \\ bÉcole Polytechnique Fédérale de Lausanne (EPFL), 1015 Lausanne, Switzerland. \\ ${ }^{c}$ KTH Royal Institute of Technology, SE-100 44 Stockholm, Sweden. \\ dTyndall National Institute, Lee Maltings Complex Dyke Parade, T12 R5CP Cork, Ireland \\ e VLC Photonics S.L., Ed. 9B, D2, UPV, Camino de vera sn, 46022 Valencia, Spain \\ ${ }^{\mathrm{f}}$ Commscope Connectivity Belgium, Diestsesteenweg 692, 3010 Kessel LO, Belgium \\ gimec vzw. 3DSIP Department, Si Photonics Group, Kapeldreef 75, 3001 Leuven, Belgium
}

\begin{abstract}
Keywords: Photonic Integrated Circuits, Silicon Photonics, Photonic MEMS, Programmable Photonics
\end{abstract}

\section{INTRODUCTION}

Photonic integrated circuits (PIC) are becoming ever more complex. This evolution is fueled by increasingly mature fabrication processes for silicon photonics ${ }^{1}$ and III-V PICs, ${ }^{2}$ as well as better photonic circuit design capabilities. ${ }^{3}$ Especially, technology platforms with a high refractive index contrast such as silicon can integrate thousands of building blocks on the same chip, boosting the level of complexity and functionality in a photonic circuit.

Today, most PICs are custom designed with one particular application in mind. The optical waveguide connections on the chip are optimized at the design stage and implemented in physical paths on the chip. These so-called application-specific photonic integrated circuits (ASPIC) have similar benefits as electronic applicationspecific ICs (ASIC) in terms of ultimate performance, efficiency and power consumption. But the development of a new ASPIC is a costly proposition, as it takes a year to design, fabricate and test a new circuit.

\subsection{Programmable PICs}

In contrast to ASPICs, the past few years have seen the emergence of programmable PICs. These photonic circuits are designed with flexibility in mind, allowing the user to configure the connectivity of the optical waveguides in the field using tunable waveguide couplers and optical phase shifters. ${ }^{4}$ Conceptually, these photonic circuits are much more like an electronic field-programmable gate array (FPGA), in the sense that the connectivity can be programmed by the user through a software interface. On the photonic chip this software programming results in electronic actuation of the optical waveguides, redirecting the light along new waveguide paths so it can be processed in real time.

Such programmable photonic circuits, which today are mostly implemented as waveguide meshes interconnected by tunable $2 \times 2$ couplers (e.g. implemented as a Mach-Zehnder interferometer), can be used to define connectivity matrices for optical routing by switching the couplers in cross or bar state. But when the tunable couplers are used in a partial coupling state, light can be routed along multiple paths, giving rise to interferometric circuits. In combination with optical phase shifters, these circuits can then project a linear combination

Further author information: (Send correspondence to W.B.)

W.B.: E-mail: wim.bogaerts@UGent.be, Telephone: 3292643324 


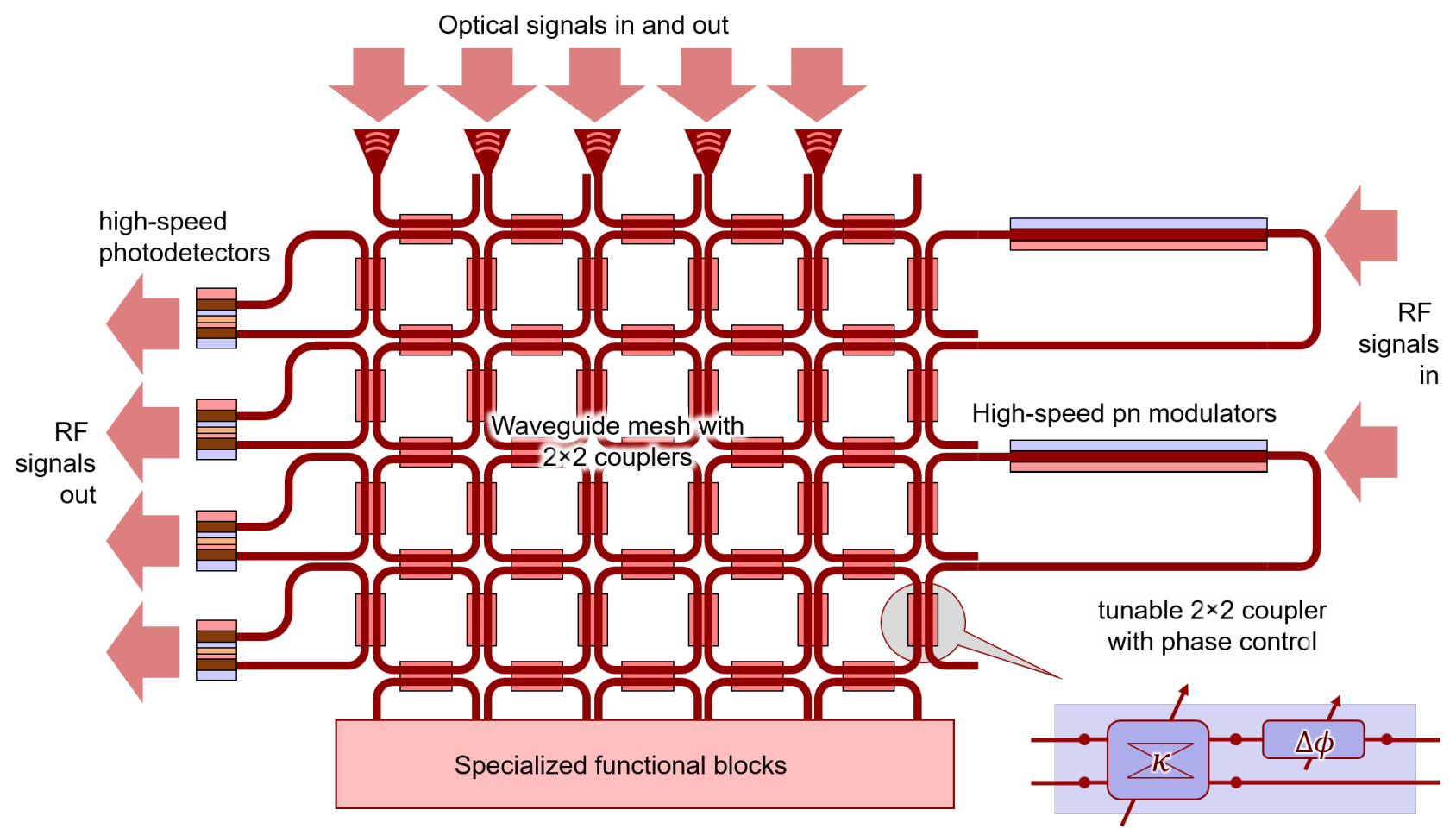

Figure 1. Possible implementation of a programmable PIC, consisting of a waveguide mesh connected to high-speed modulators, photodetectors, optical interfaces and specialized high-performance blocks.

of input ports onto a set of output ports, ${ }^{5,6}$ effectively performing a matrix-vector multiplication. Such operations are very useful for a variety of applications such as artificial neural networks ${ }^{7,8}$ and quantum information processing. ${ }^{9-12}$ Also, when incorporating feedback loops and delay lines in the waveguide mesh, it becomes possible to define programmable optical filter circuits which could be useful for applications such as microwave photonics. ${ }^{13-15}$ But like electronic FPGAs, programmable photonic circuits can outgrow specific applications. In combination with high-performance building blocks such as high-speed electro-optic modulators (e.g. implemented using p-n junctions, ${ }^{16}$ by electro-absorption ${ }^{17}$ or other mechanisms), optical amplifiers, high-speed detectors, nonlinear elements of long delay lines, they can be programmed to perform a variety of functions. ${ }^{18}$ Even though their performance would not be as good as that of a dedicated PIC, the off-the-shelf availability and real-time programmability might make them economically attractive for many applications that do not warrant the cost of developing a custom ASPIC.

\subsection{Challenges for Programmable PICs}

Programmable PICs come with a number of challenges. The first is raw performance: because the circuit consists of a configurable waveguide mesh connected with many programmable $2 \times 2$ couplers, this will automatically induce higher optical losses because of the longer optical paths and larger numbers of components to traverse. Today, most optical phase shifter implementations come with some drawback: Thermal phase shifters (heaters) consume a lot of power, ${ }^{19}$ carrier-based phase shifters have a high optical insertion loss ${ }^{20}$ and the same is true for phase shifters based on materials like liquid crystals. ${ }^{21}$ Low-loss phase shifters based on the Pockels effect are typically weak and require a long path length. ${ }^{22}$ It is also possible to use micro-electromechanical systems (MEMS) in combination with optical waveguides to implement low-power couplers and phase shifters. ${ }^{23}$ Most of these phase shifter technologies either require constant application of electrical power (heaters, carrier injection) or a voltage (carrier depletion, Pockels effect, liquid crystals), although there have been demonstrations of nonvolatile electro-optical actuators (mostly switches) based on on phase change materials ${ }^{24}$ or MEMS. ${ }^{25}$ 
A second challenge relates to the design of the programmable circuit topology itself. Today, most programmable circuits are implemented as either an interferometric mesh where light propagates in one direction (a "forward-only" mesh ${ }^{6,7,10}$ ), or a uniform mesh of square, triangular or hexagonal cells where light can be routed in loops (a so-called "recirculating" mesh ${ }^{14,15}$ ). While these have been proven on a limited scale, it is not proven that these mesh topologies are best suited for scaling up programmable PICs to truly generic photonic chips.

A third challenge comes with the complexity and control of the circuit. A large waveguide mesh can easily require hundreds (or even thousands) of electrical drivers, and these need to be independently controlled. This requires close integration with electronics, and in many cases also the incorporation of monitor detectors inside the optical circuit. ${ }^{6,26}$ This immediately translates into a packaging problem to interface photonics and electronics, and it becomes even more challenging if high-speed electrical input/outputs are needed for microwave photonics functions.

Finally, there is an open challenge in the "interfaces" to a programmable PIC. A user of a programmable photonic circuit should be able to access the functionality within the chip, and have an application programming interface (API) to configure the chip to perform the desired functions. Such an API can consist of several software abstraction layers, but eventually needs to interface to the hardware through several layers of digital and analog electronics.

\subsection{The MORPHIC Project}

The European Horizon 2020 project MORPHIC (Mems-based zerO-power Reconfigurable PHotonic ICs) is working on those challenges for programmable PICs. Running from 2018 till 2021, the 6 partners of this vertically integrated project bring together expertise in silicon photonics, photonic MEMS, photonic-electronic packaging, circuit design and different application spaces (www.h2020morphic.eu).

As shown in Fig. 2, the core technology being developed in MORPHIC is an implementation of compact, low-power electro-optic phase shifters and tunable couplers based on waveguide MEMS. By mechanically moving a waveguide core closer or further from neighboring waveguides, a strong phase shift can be induced by electrostatic actuation. ${ }^{23}$ Waveguide MEMS phase shifters, ${ }^{27-30}$ tunable couplers ${ }^{31}$ and switches ${ }^{25,32,33}$ have already been demonstrated, but where MORPHIC differs is in the integration of such photonic MEMS actuators in an established fully functional silicon photonics platform, namely imec's iSiPP50G platform, ${ }^{34}$ which already contains high-quality passive waveguides as well as high-speed modulators and photodetectors. This integration is done without modifying the process flow of the silicon photonics platform, but by only adding a few post-processing steps. The challenge in building these MEMS-based components is to keep the optical losses sufficiently low, the footprint compact, and not disrupting the performance of the other elements in the silicon photonics platform.

These MEMS-based phase shifters and couplers are then integrated into larger circuits, both applicationspecific circuits for switching, optical beamforming or microwave processing, as well as generic waveguide meshes where tens or hundreds of MEMS actuators are connected into a programmable circuit. For this, we are exploring how different circuit topologies can trade-off flexibility for performance, especially as we scale up these large circuits.

Interfacing these large circuits with the outside world and their control electronics is an integral part of the project. The large photonic MEMS circuits require hermetic sealing, hundreds to thousands of low-speed electrical control lines, and tens of optical and high-speed radio-frequency (RF) signal lines. The MORPHIC project is developing a generic interposer-based technology that can handle these large numbers of connections.

The physical interfaces are only part of the system. We are also developing the driver electronics for the many MEMS actuators, the readout circuits for built-in monitor photodiodes, and configuration and software layers that will allow a programmer to configure the circuits for different applications.

We are demonstrating the technology on three different application scenarios: Large optical switch matrices, optical beamforming networks, and a microwave photonics processor. For each of these applications, we are benchmarking how the MEMS-based phase shifters and couplers benefit the performance of an optimized ASPIC, but we also test how the same functionality performs when it is programmed into a generic programmable PIC.

In this paper, we look in more depth into these different aspects of the MORPHIC project, and discuss a number of intermediate accomplishments in the first half of the project. 


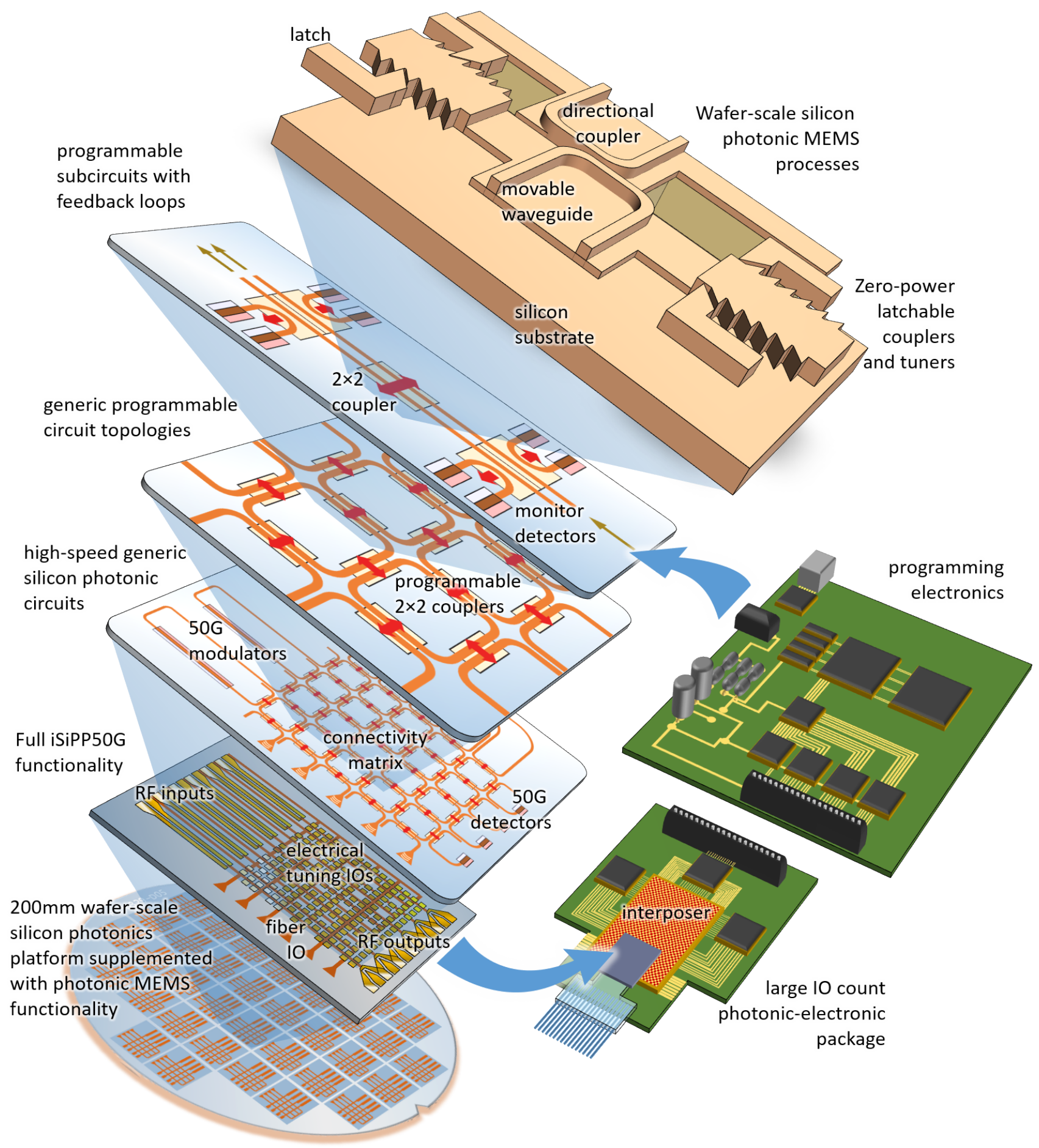

Figure 2. The MORPHIC project brings together silicon photonics process technology, photonic MEMS device design, programmable circuits, packaging, driver electronics and software to demonstrate the versatility of programmable PICs in different application scenarios. 

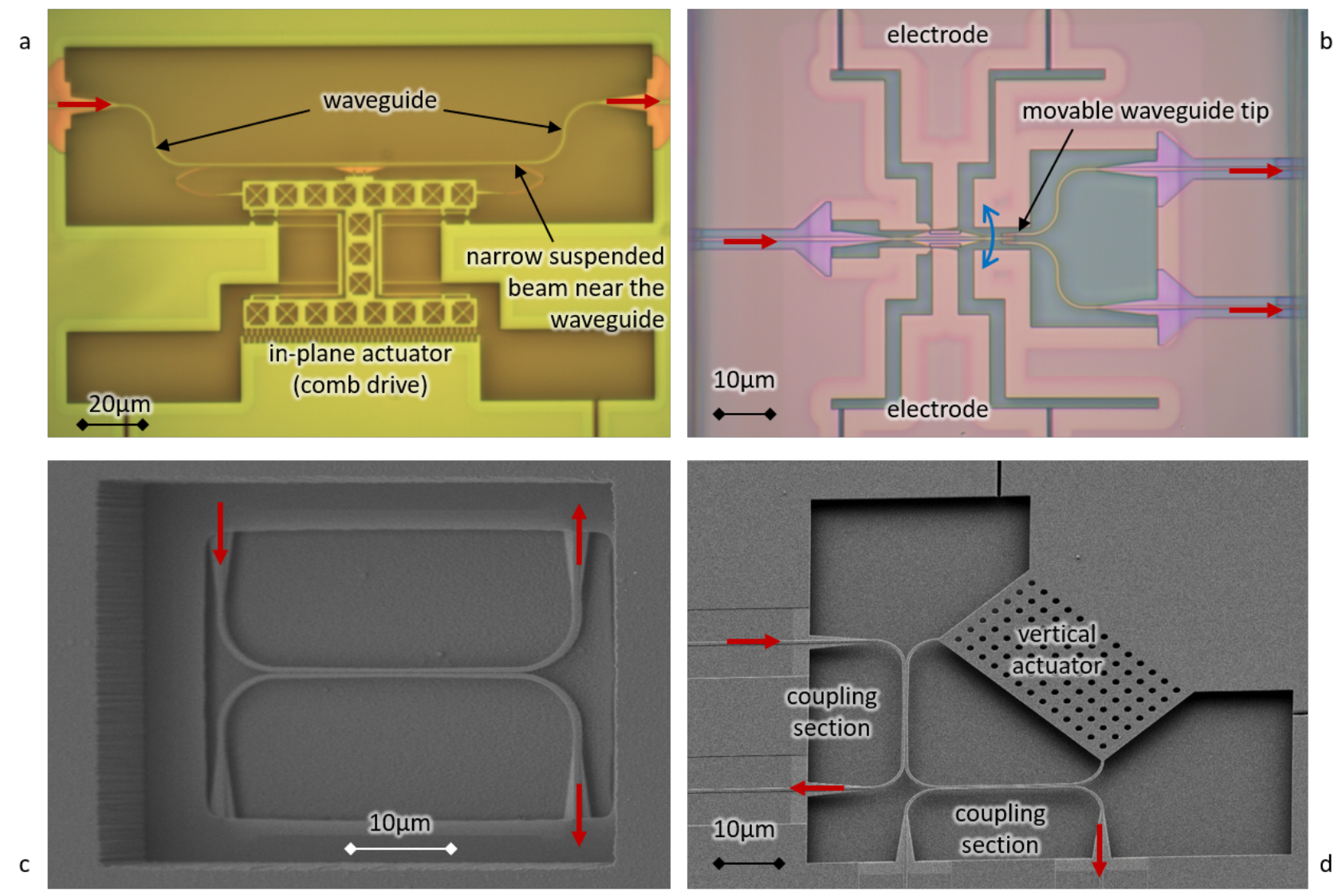

Figure 3. Different silicon photonic MEMS structures in MORPHIC. (a) Tunable optical phase shifter using an inplane comb-drive actuator, (b) $1 \times 2$ switch using a single waveguide tip that can be coupled to two output tips, (c) a suspended directional coupler with broadband wavelength transmission, ${ }^{35,36}$ (d) vertically actuated $1 \times 2$ switch with tolerant directional couplers.

\section{PHOTONIC MEMS ACTUATORS}

In MORPHIC, we are developing electro-optic phase shifters and tunable $2 \times 2$ couplers based on microelectromechanical structures embedded in the waveguide structures. The operational principles of MEMS have a number of benefits compared to other electro-optic actuation mechanisms: when properly engineered, they do not introduce additional optical transmission losses as the materials used are not absorbing. They can be actuated electrostatically, which implies that there is no static power consumption, and when they are sufficiently stiff they can switch on sub-ms time scales. ${ }^{23}$

The most commonplace mechanism to implement an electrically actuated optical phase shift in a silicon waveguide is through thermal tuners, in the form of heaters that are embedded inside or close to the waveguide core. As silicon has a high thermo-optic coefficient, the heaters can be quite compact. However, the constant generation of heat requires continuous electrical power consumption, usually of the order of 10-20 $\mathrm{mW}$ to induce a $\pi$ optical phase shift. Still, heaters are used often because of their simplicity and because they induce little or no optical transmission loss.

Other mechanisms, such as carrier-induced phase shifters (also called plasma-dispersion phase modulators), introduce an inherent optical loss because free carriers also absorb light. It is also possible to incorporate additional materials that can be electrostatically actuated, but the effect is weak or the material introduces additional optical losses (e.g. scattering in liquid crystals). 


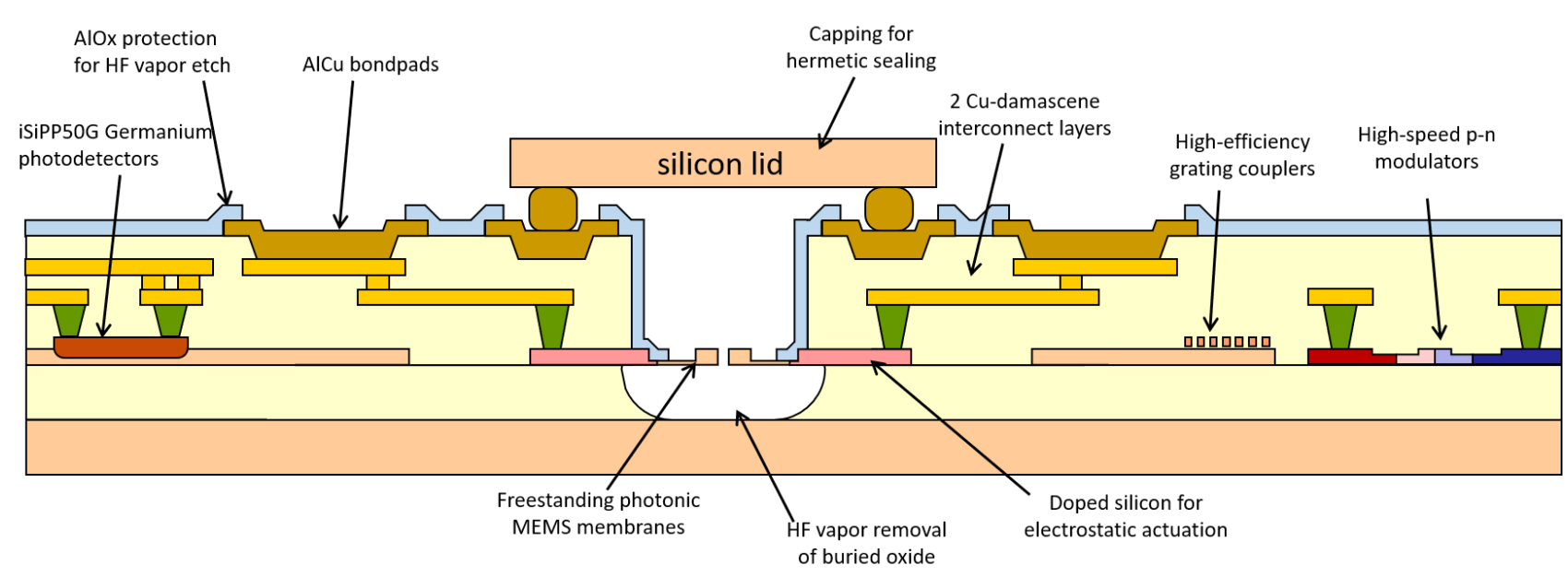

Figure 4. Cross section diagram of the iSiPP50G platform, ${ }^{34}$ with the extensions for photonic MEMS.

\subsection{Photonic MEMS actuation mechanisms}

MEMS electro-optic actuators make use of moving parts to change the optical properties of a waveguide. For instance, as shown in Fig. 3 to induce a phase shift, a narrow rim of high-index material can be moved closer to or further from the core of the waveguide, changing the effective index of the guided mode. ${ }^{37}$ When the transition geometry is properly engineered, such a 'loading' structure does not induce scattering, leakage or absorption beyond what is already present in the waveguide itself. Likewise, a tunable $2 \times 2$ coupler can be implemented by two parallel waveguide cores (like the suspended directional coupler shown in Fig. 3c), where the separation between the waveguide is mechanically controlled. ${ }^{36}$

We can separate the movement of the waveguides into two classes, based on how they are electrostatically actuated, as shown in Fig. 3. Waveguide cores or loading structures can be moved vertically by applying a voltage over different layers or the silicon substrate, bending a cantilever structure upward or downward. ${ }^{29}$ Alternatively, a suspended beam can be shifted in plane through comb-drive actuators. ${ }^{37}$ In both cases, the actuation is electrostatic, by applying a voltage to induce an attractive force between a movable and fixed element. Alternative methods to induce a movement is through thermal expansion or piezo-electric effects, but these are not the primary exploration path in MORPHIC. However, to reduce the reliance on always-on electronics, we are exploring mechanical bistable and latching mechanisms. ${ }^{31,38}$

\subsection{Processing Silicon Photonic MEMS}

Photonic MEMS require free-standing geometries that can move around either vertically or in the plane of the chip. However, most silicon photonics technologies today encapsulate the silicon waveguides in a material stack of silicon dioxide, which also contains the layers of metal wiring to connect the active components in the waveguide layers, such as modulators and photodetectors. ${ }^{34}$ So a MEMS-enabled silicon photonics process imposes requirements that are on first sight incompatible with established platforms. To reconciliate these requirements, three approaches can be considered:

- Building a new platform from scratch: In the long term this can lead to the best performance, but it would require not just the development of the MEMS technology, but also the complete redevelopment of the active components such as modulators and detectors.

- Inserting a module in the existing process: We could try adding MEMS functionality during the processing of the waveguides. As the waveguide processing is quite early in the flow, the additional module could impact the later modules for the active components. This is especially true for the MEMS devices, as they require free-standing components. 

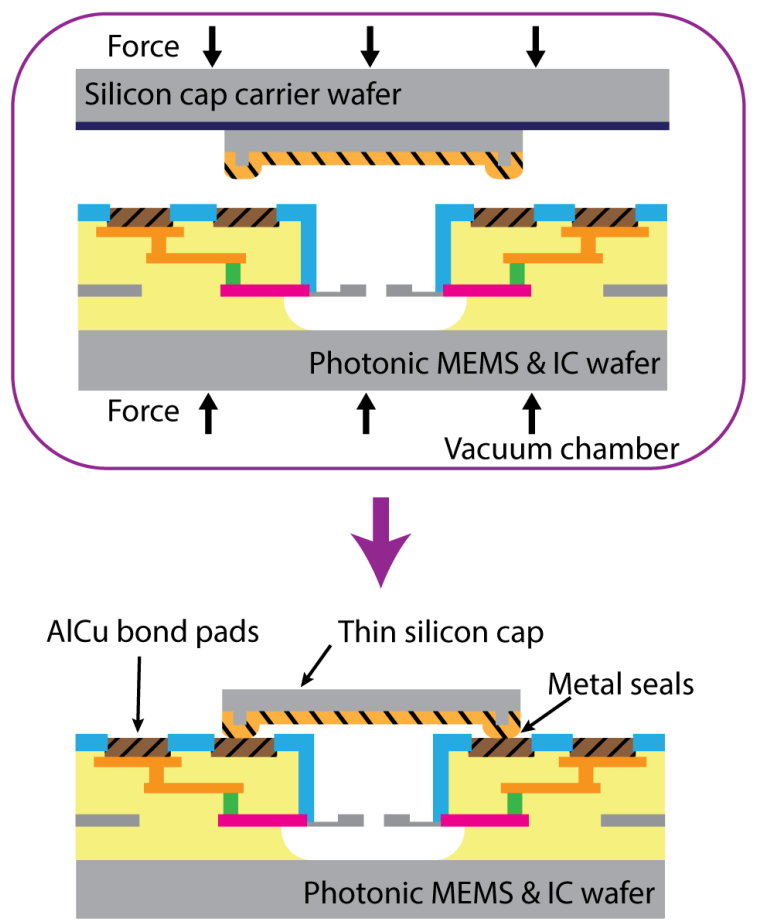

b

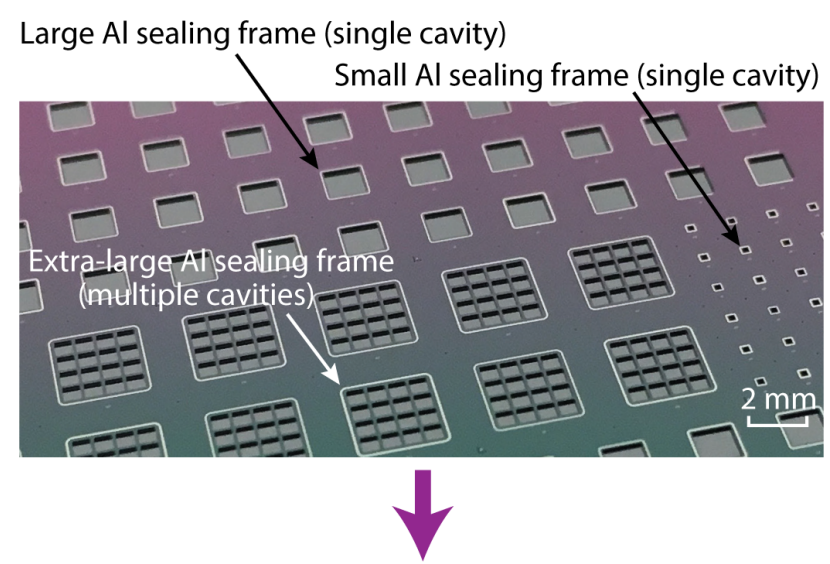

Transferred and sealed large /extra-large /small thin Si caps

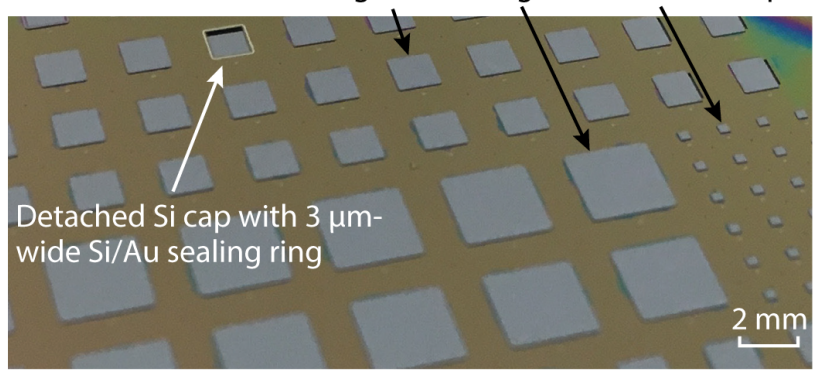

Figure 5. Wafer-scale sealing process to be used for the silicon photonic MEMS in MORPHIC. (a) Proposed method for hermetic sealing of Photonic MEMS wafers using wafer bonding and transferred thin silicon caps. (b) Demonstration of the sealing method on a dummy wafer with empty cavities. ${ }^{39}$

- Post-processing the MEMS on the platform: Rather than changing the established platform with its optimized flow and process monitoring, we can use the existing geometry layers for waveguides to define the MEMS structures, and use a few post-processing modules to open up the MEMS cavities and release the free-standing waveguides from the substrate.

In MORPHIC we opted for the third option, which imposes fewer restrictions on the temperature budget and material contamination of the MEMS processing. This allowed us to integrate the MEMS fabrication with imec's established iSiPP50G process. The result is illustrated in Fig. 4. The iSiPP50G process starts from a $220 \mathrm{~nm}$ silicon-on-insulator (SOI) layer on a $2 \mu \mathrm{m}$ buried oxide. The passive structures are defined using three levels of etching, either fully etching the silicon, or partially etching it either $70 \mathrm{~nm}$ or $150 \mathrm{~nm}$ deep. An additional poly-silicon overlay is added to define high-efficiency grating couplers. Then, several implantation steps define doping levels for carrier-based p-n modulators, and germanium epitaxy is used to integrate efficient high-speed photodetectors. The back-end electrical connections are defined in a two-level copper-damascene process finished by $\mathrm{AlCu}$ bondpads.

For the MEMS processing, we make use of an existing module at the end of the iSiPP50G process flow which opens up a cavity in the back-end stack down to the level of the waveguides. This module, which is originally developed for sensor applications, exposes the silicon waveguides. To release the waveguides from the underlying buried oxide, we first cover the rest of the chip with an alumina ( $\mathrm{AlOx}$ ) protection layer deposited with atomic layer deposition (ALD), and patterned with direct-write maskless optical lithography. Using a vapour etch with hydrofluoric acid (vHF), we then selectively remove the oxide next to and underneath the silicon waveguides. The result is a free-standing silicon waveguide. 

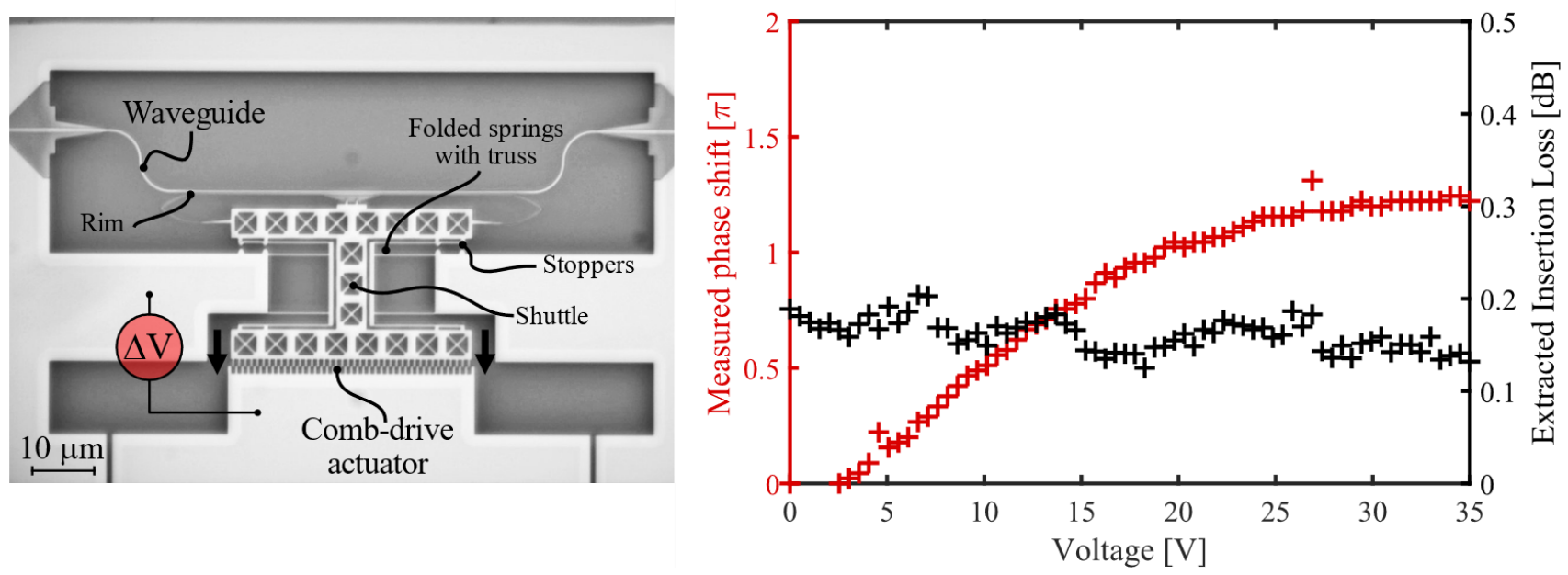

Figure 6. Example of MEMS phase shifter using horizontal actuation with comb-drives. The device was measured on an early chip from the second MORPHIC fabrication run. In a), optical microscope view of the phase shifter, and in b), corresponding measured phase shift and insertion loss against voltage, at $\lambda=1550 \mathrm{~nm}$.

\subsection{Sealing the MEMS}

The free-standing MEMS are exposed to the environment. To ensure reproducibility and reduce the effects of external operational factors such as atmosphere composition and humidity, the MEMS cavities need to be sealed. In MORPHIC, we add hermetically sealed thin caps to the MEMS cavities with a wafer-level bonding and cap transfer process, using the metal bond-pads present on the silicon photonics wafers as a landing surface. ${ }^{39}$ Fig. 5 shows a part of a wafer having MEMS cavities with caps of different sizes.

\subsection{Silicon photonic MEMS phase shifters}

In the first two fabrication runs of the MORPHIC project we prototyped a variety of silicon photonic phase shifters. Fig. 6 shows an example of device that relies on in-plane comb-drive actuation to increase the gap between a fixed waveguide and movable narrow rim. This modification of the waveguide cross-section results in a decrease of the effective index, and with an interaction length of $50 \mu \mathrm{m}$, to a significant phase shift. This device was measured on an early chip from the second fabrication run, which did not include any of the metal layers from iSiPP50G. It was probed using the silicon anchor pads, see Fig. 6a. Our phase shifter is compact, has a passive insertion loss of only $0.2 \mathrm{~dB}$, and attains linear phase shifts up to $\pi$ with only $20 \mathrm{~V}$ actuation voltage, see Fig. $6 \mathrm{~b}$.

\subsection{Silicon photonic MEMS couplers and switches}

Similarly, we also implemented MEMS devices for optical power distribution on the chip, such as $1 \times 2$ and $2 \times 2$ tunable couplers and switches. For example, a $1 \times 2$ tip-to-tip switch provides a compact photonic MEMS switch which. The device is a photonic analogous to electronic Single-Pole Double-Throw (SPDT) switches. It consists one movable and two fixed waveguide tips. The movable tip is actuated by a pair of curved electrostatic actuators. In the OFF state of the switch $(0 \mathrm{~V})$ the component is lossy, and ideally the residual optical power is divided equally between two ports as the movable tip rests between the fixed tips with an equal distance from them. By applying voltage to one of the electrodes, depending on which electrode is actuated, the waveguide tip moves towards one fixed tip and optical power transfers to the corresponding port, suppressing the power in the other port. This particular device exhibits an extinction ratio of $25 \mathrm{~dB}$. The device has a compact footprint of only $30 \mu m \times 60 \mu m$. 

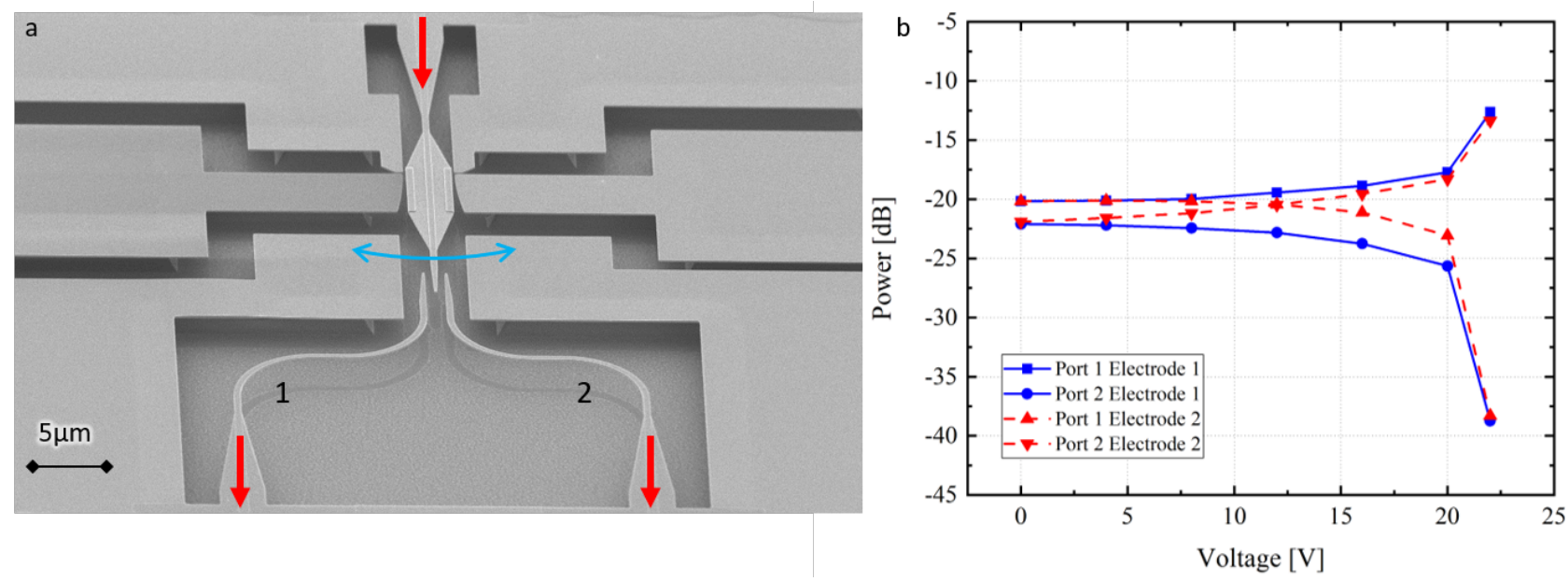

Figure 7. Tip-to-tip optical waveguide switch from Fig. 3b. The waveguide tip of the input can be moved electrostatically towards one of the output tips. (a) SEM image of the tip-to-tip switch. (b) Transmission power (fiber to fiber) at $1550 \mathrm{~nm}$ in the two outputs when addressing one or the other electrode.

\subsection{Integration challenges for MEMS components into circuits}

While single silicon photonic MEMS actuators have already been demonstrated, ${ }^{23,40,41}$ they only become truly useful if they can be connected into circuits and combined with the other functional components in a silicon photonic chip. This integration imposes some boundary conditions. First of all, the MEMS components are surrounded by air or vacuum, while the active components and other waveguides are surrounded by a dielectric cladding consisting mostly of silicon dioxide with a refractive index of 1.45. This means that the waveguides need to transition between the two types of cladding every time a phase shifter or tunable coupler is needed. Designing a compact, low-loss, low-reflection interface between these two claddings is essential to make MEMS actuators useful at the circuit scale. When combining multiple MEMS actuators in a circuit, we need to consider whether to combine multiple MEMS elements together in the same cavity to reduce the number of oxide/air transitions.

As the MEMS actuators are electrostatically controlled, it is important that the electrical driving signals of different MEMS components do not affect one another. This means that two MEMS components need to be electrically insulated from each other, or that any galvanic connection between the two is always at the same potential (e.g. grounded). As silicon waveguides are conducting, this requires that either all waveguides are at ground potential, or that the waveguide core needs to be cut in strategic places to apply a galvanic insulator. This latter is bound to induce additional losses in high-contrast silicon waveguides, while the first condition imposes severe limitations on the type of MEMS geometries that are possible in the platform.

\section{PROGRAMMABLE CIRCUITS}

To realize programmable circuits in MORPHIC, we need to bring MEMS components together into a large generic mesh, and electrically connect it so we can address all the individual elements. In programmable photonic waveguide circuits, we can discern two large classes of waveguide meshes: forward only and recirculating meshes. A forward only mesh, as illustrated in Fig. $8 \mathrm{a}^{5,6,10,11}$ has light propagating in a single direction through the mesh, by connecting $2 \times 2$ couplers and phase shifters in a left-to-right topology. Such meshes are particularly useful for functions that require linear matrix operations, such as quantum information processing or artificial neural networks. ${ }^{7,8,10,12}$ However, when we need a generic circuit to connect functional elements together or implement spectral filter functions, these meshes are not sufficiently flexible.

Therefore, in MORPHIC we look more into the second class of recirculating waveguide meshes, as shown in Fig. 8b-d. By organizing waveguides in loops connected with programmable $2 \times 2$ couplers, we can direct light from any waveguide port to any other waveguide port. Light can now be routed along discrete delays to 

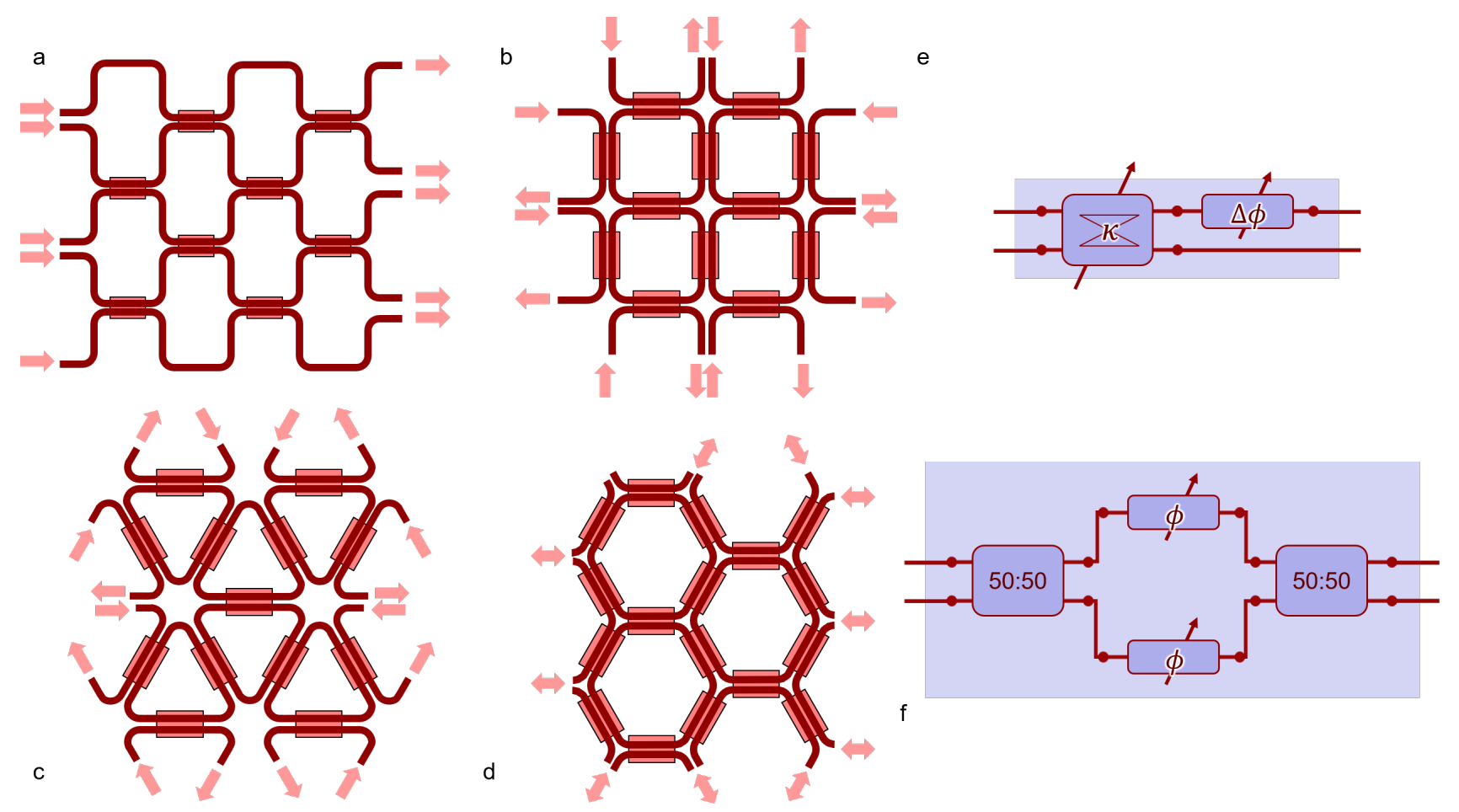

Figure 8. Programmable waveguide meshes. (a) Forward only ${ }^{5,6,10,11,42}$ and (b-d) recirculating waveguide meshes with different cell shapes. ${ }^{15,43}$ To implement the basic $2 \times 2$ element on the mesh edges, we can either use (e) a tunable $2 \times 2$ power coupler with an additional differential phase shifter, or (f) a Mach-Zehnder interferometer with two phase shifters that can be driven independently in either differential and common mode.

implement finite impulse response filters, and even in ring resonators to implement infinite impulse response filters. ${ }^{14}$

There are different ways to organize the waveguides in a recirculating mesh. Regular meshes can be square, ${ }^{15}$ triangular or hexagonal. ${ }^{43}$ Especially this latter geometry, shown in Fig. 8d, is very flexible in the delay increments that can be implemented, and it also allows a coupling between clockwise and counterclockwise circulating light.

For all these meshes, it is important that the length of an edge (called basic unit length in ${ }^{14,43}$ ) is as short as possible, as this determines the free spectral range of any filter that can be programmed into the mesh. The MEMS components in MORPHIC can help here, as they can apply a strong phase shift or coupling along a short optical length.

\subsection{MEMS-based subcircuits}

For a first generation programmable circuit, we opted for a hexagonal recirculating mesh topology ${ }^{14,44}$ using tunable MEMS directional couplers connected with phase shifters on three corners in each hexagon cell, while the connections for the other three corners are waveguides. This is illustrated in Fig. 9. To minimize transition losses within a unit cell, three couplers and three phase shifters are grouped together in a single MEMS cavity. Then, multiple of these cavities are connected by oxide-clad waveguides. At the time of writing, we have not yet performed measurements on these circuits.

\subsection{Subcircuits with Active Monitoring}

In larger circuits, we cannot expect all actuators to behave identically. There is variability from fabrication processes, but also the driver circuits and use of the elements can be a cause for different behavior. While some of this variability can be compensated by regular calibration routines, it is often useful to monitor the behavior in real time. For this, MORPHIC is developing subcircuits which include built-in monitors, as shown in Fig. 10. 

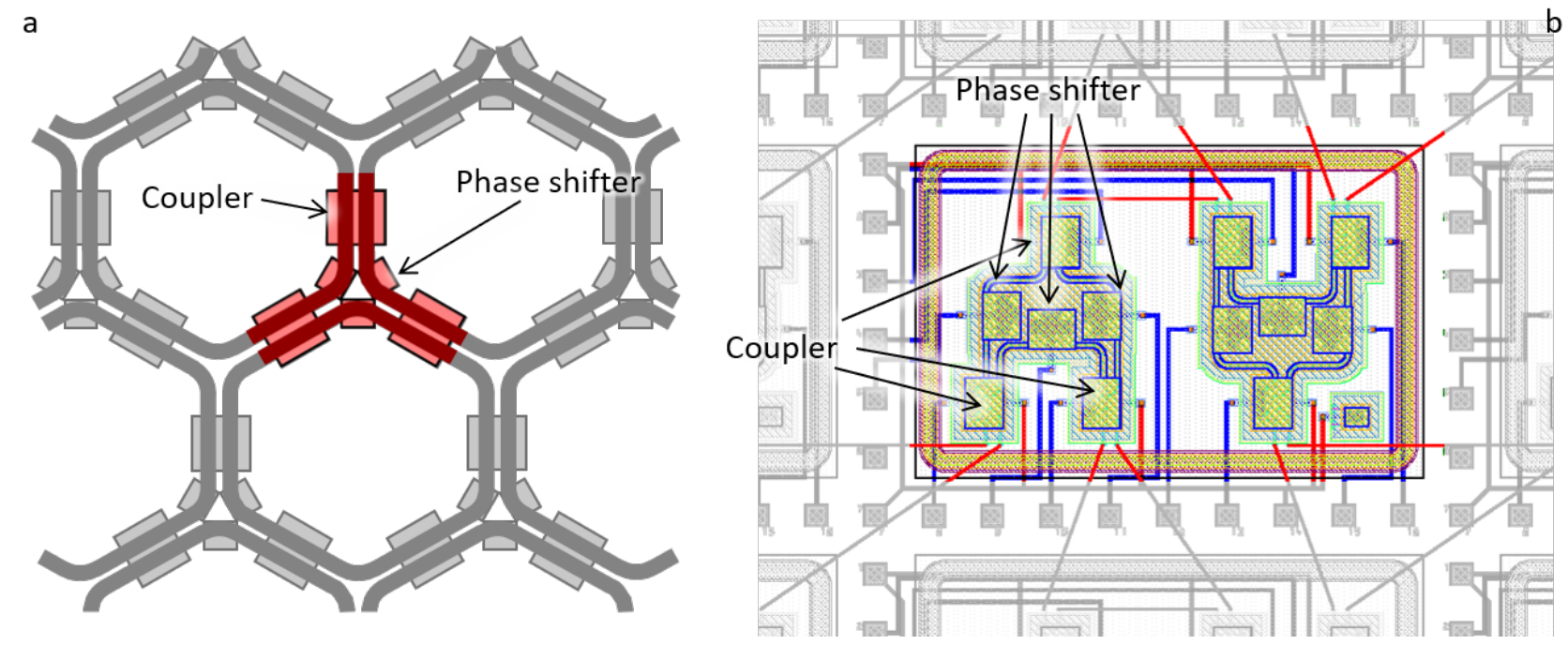

Figure 9. MEMS subcircuit implementation for a generic programmable Photonic hexagonal mesh. (a) Unit cell consisting of 3 tunable couplers and 3 phase shifters. (b) mask implementation of the unit cell.
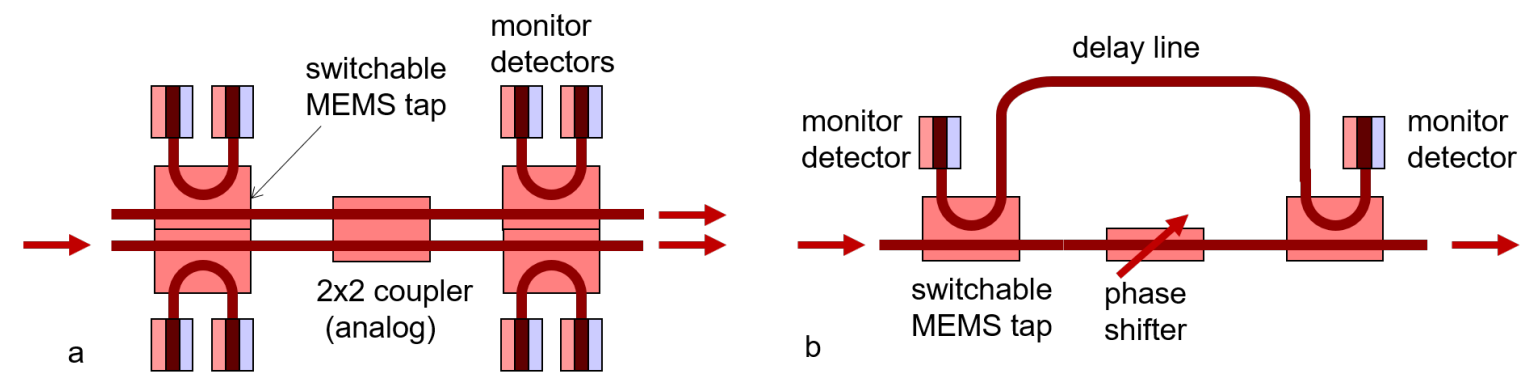

Figure 10. Subcircuits for (a) tunable couplers and (b) phase shifters with built-in monitors. The monitors can be activated using a MEMS switch that implements a power tap on the waveguide.

The phase change induced by a phase shifter can be monitored by an additional interferometer with (balanced) photodiodes, while the power coupling in a tunable coupler can be monitored using integrated photodetectors or other monitoring mechanisms such as contactless probes. ${ }^{26}$ The advantage of the MORPHIC MEMS devices is that we can design these monitoring circuits in such a way that they can be physically moved away from the main optical waveguide path, such that there is no longer any perturbation of the waveguide mode. This way, it becomes possible to use the monitors during configuration, but then disconnect them to reduce the impact on optical transmission losses during operation.

\subsection{Circuit Configuration and Parasitics}

One of the challenges with generic programmable circuits, is that not all components will effectively perform as intended, and the variation in bulding blocks can have a larger impact. In a custom-designed ASPIC, a small change in coupling coefficients or phase shifts along the path of the light can induce additional transmission loss, or a mismatch in extinction ratio of an optical filter. In a programmable circuit, the light has to pass through many more phase shifters and couplers, which all will induce some imperfection. This will aggravate the performance penalty due to these parasitics.

But in programmable waveguide meshes these imperfections induce another problem: errors in the coupling ratios of the tunable couplers will route light along alternative paths through the mesh. These paths can eventually recombine with the main path of light, interfering either constructively or destructively depending on 
the phase shifters along the path and the optical path length difference. ${ }^{45,46}$ This is shown in Fig. 11, where the transmission fluctuations as a function of wavelength are plotted for different variations in the coupling coefficients of all the couplers in the mesh. As the route through the mesh gets longer, the fluctuations increase, aggravating the interferences of the parasitic paths.

The effect of the parasitics can be influenced and mitigated by tuning the couplers that are not actively used in the optical path. By setting the unused couplers in cross state, leaking light is shunted away from the main path and it no longer gets the opportunity to accumulate in parasitic ring resonators. While this does not improve losses due to parasitic coupling, it significantly suppresses the wavelength-dependent fluctuations.

\section{PACKAGING, DRIVERS AND INTERFACES}

Programmable photonics is more than the photonics chip. Because of the large number of electro-optic actuators, a programmable PIC cannot be considered separate from its control electronics. The hundreds or even thousands of actuators and monitors require connections between the photonics and the electronics, which, if the electronics is not integrated on the same chip, translates into a packaging and assembly challenge. ${ }^{47}$ Add to that the constraints of hermetically sealing the MEMS actuators, the interfacing with several (tens of) optical fibers, as well as high-speed RF input and output signal, and it becomes clear that packaging cannot be treated as an afterthought in programmable photonics.

\subsection{Packaging Strategy}

In MORPHIC we have chosen to keep the electronics and photonics separate, and use a packaging strategy to bring the two together. The packaging concept is shown in Fig. 12. Because we want to bring many hundreds of electrical connections off the photonic chip, we cannot rely on simple wire-bonding along the perimeter of the chip, but we need to use the entire chip surface for electrical connections. We chose an interposer-based approach. We predefined 3,300 bondpad locations on a sparse grid on the photonic chip, so we can use a single high-density, multi-layer, ceramic interposer to fan out these 3,300 bondpads to a printed circuit board that

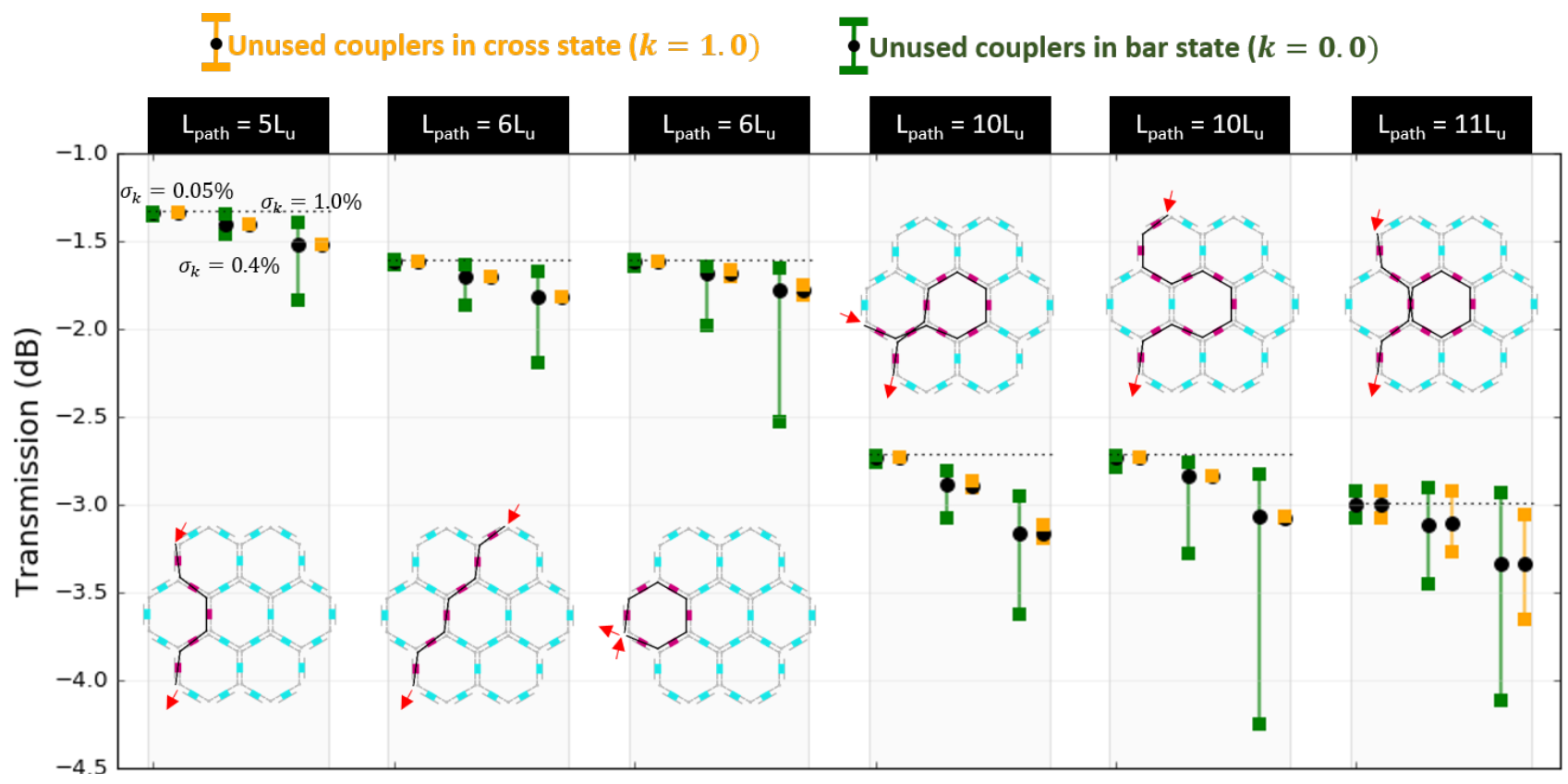

Figure 11. Effect of parasitics in programmable circuits. The error bars indicate fluctuations in the transmission due to random errors in the tunable coupler settings. The green bars are the errors when the unused couplers are in bar state, while the yellow bards are the errors when the couplers are in cross state. The errors become more pronounced when the route through the mesh becomes longer. ${ }^{45}$ 

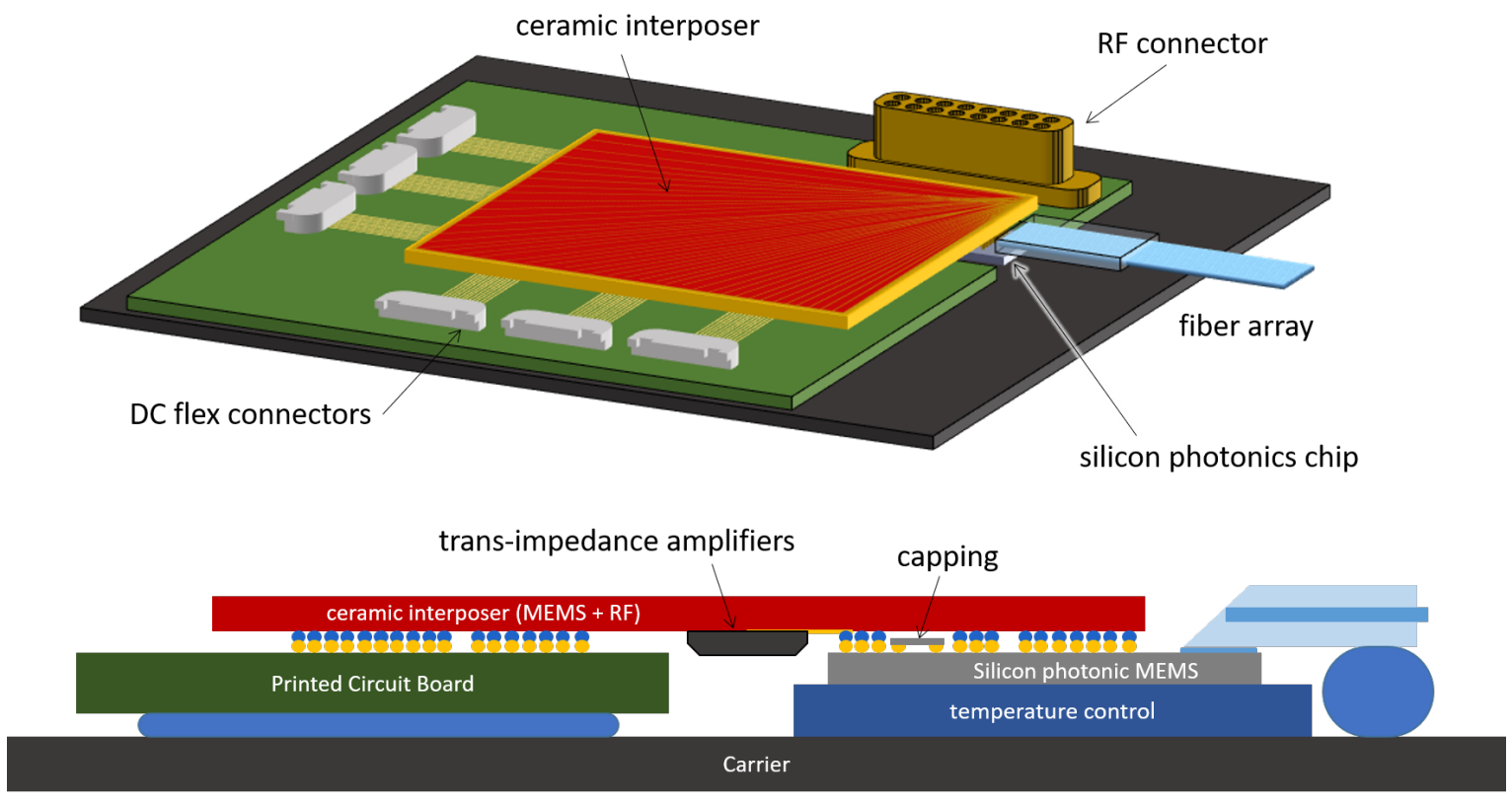

Figure 12. Packaging and assembly strategy for electrical, optical and high-speed RF interfaces to the silicon photonics programmable PIC.

then connects with the driver and readout electronics. The interposer also supports high-frequency RF strip lines for the $12 \mathrm{RF}$ inputs (modulators) and $12 \mathrm{RF}$ outputs (balanced photodetectors). The latter also require trans-impedance amplifiers packaged as closely as possible to the photodetectors. ${ }^{48}$

The mounting of the silicon photonics chip on the interposer needs to be compatible with the MEMS sealing process discussed earlier. The bonded caps over the MEMS cavities are several tens of micrometers thick, and the electrical connections (i.e. microbumps) need to leave sufficient margin to make a good ohmic contact.

For the optical interfaces, we routed the on-chip waveguides to one edge of the chip, where we can connect up to 72 optical fibers in a single array, which can be mounted in-plane with an angle-polished facet to interface with the on-chip grating couplers. ${ }^{49}$

\subsection{Control Stack}

To interface the programmable PIC with the user, a control stack is needed, consisting of analog and digital electronics, as well as several software layers with different levels of abstraction. This is illustrated in Fig. 13.

For the driver electronics we chose to use off-the-shelf electronics as much as possible. While a custom, driver ASIC is definitely the more scalable solution in the long term, the unknown aspects of the silicon photonics MEMS devices and the packaging strategy makes the design of an ASIC a risky and costly proposition.

Electrostatic driving of MEMS devices typically requires high voltages, between $20 \mathrm{~V}$ and $100 \mathrm{~V}$. For the MORPHIC devices, we opted to limit the driving to $50 \mathrm{~V}$, using 14-bit digital-to-analog converters (DAC). Of course, the packaging materials and interposer need to be compatible with this driving voltage. Choosing a suitable DAC with sufficient resolution and voltage range is important, as DAC discretization errors can contribute to the parasitic paths in a programmable photonic circuit. ${ }^{50}$ For the readout of monitor photodetectors, we use a 16-bit analog-to-digital (ADC) converters.

To control the large number of analog drivers, we will construct a modular system controlled by a hierarchical digital network, that in turn can be programmed from a computer.

To complement the hardware, we are also building the necessary software layers that control the various aspects of the programmable chip. At the lowest level, there are feedback control routines that use the readout 


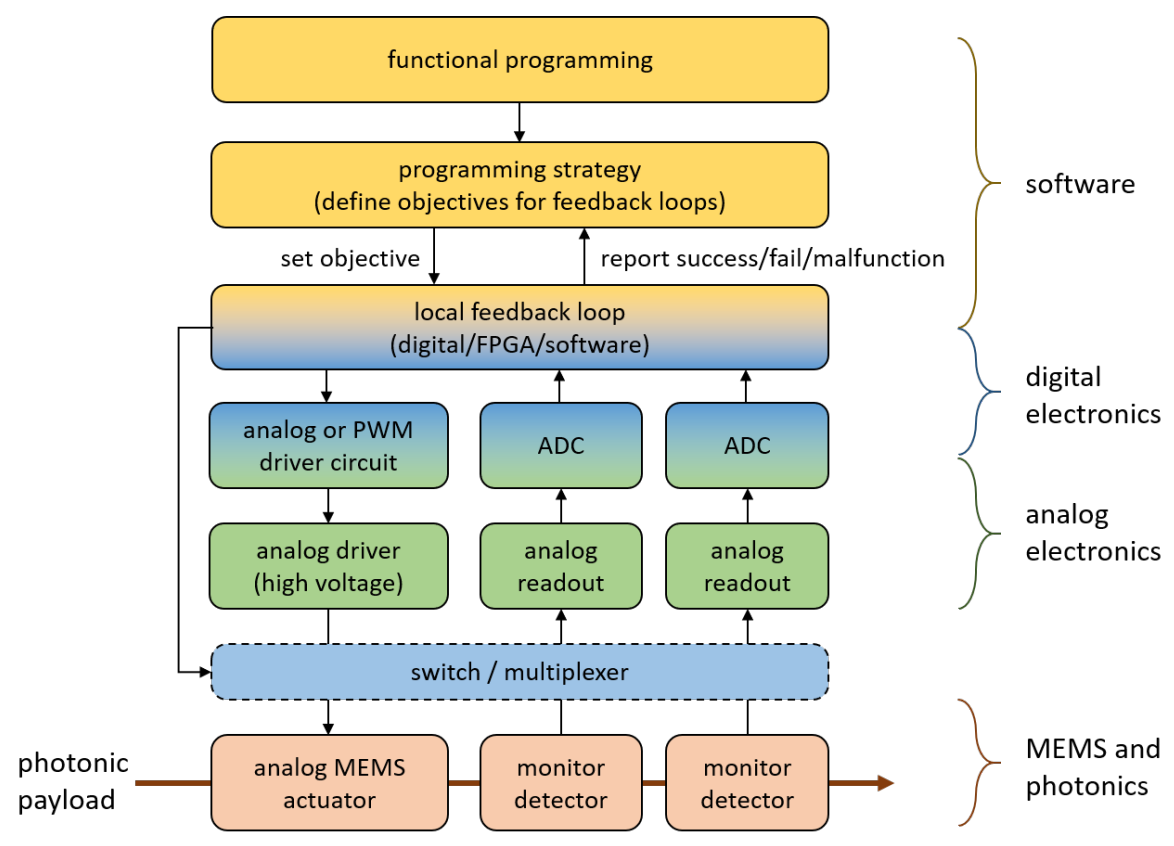

Figure 13. Control and software layers.

of the on-chip monitors to stabilize the MEMS actuators to their desired state. For certain configurations, these can be very simple minimizing or maximizing routines. ${ }^{6}$ At a higher level, programming routines are needed to help the designer implement functionality in the chip. One possible approach is the use of graph representations of the photonic circuit to route or distribute light on the chip. ${ }^{51,52}$ Another functional layer where MORPHIC is working on is the synthesis of filter circuits in a programmable waveguide mesh. These software layers need an application programming interface for a circuit designer to design and test functionality in such a programmable circuit.

\section{DEMONSTRATORS}

In MORPHIC, we want to apply the vertical technology stack (silicon photonic MEMS processing, device design, circuits, packaging, electronics and software interfaces) to a number of specific applications. In particular, we want to prove that the technology is both valuable for ASPICs as for generic programmable photonic circuits. Therefore, we chose to build demonstrators for three distinct applications using the same technology

- Optical Switch Matrices: using the low-power, or even the non-volatile MEMS components, we can build large switch matrices for different kinds of optical networks. We intend to demonstrate the MORPHIC technology with a $16 \times 16$ switch matrix.

- Optical Beamforming Networks: Using a combination of tunable couplers and phase shifters we can distribute light over a large number of optical 'antennas' to control the shape and direction of a narrow optical beam in the far field. This is useful for applications in free-space communication and LiDAR. We intend to demonstrate the MORPHIC technology in a small-scale 32-antenna optical phased array.

- Microwave Processing: Using the high-speed modulators and fast photodetectors, we can encode an RF signal on an optical carrier and manipulate its spectrum using a programmable optical filter. This can be used to clean up RF signals (e.g equalization) or filter out a specific frequency channel. In this application space, we want to build a 4-channel microwave processor.

For each of these applications, we will build an optimized silicon photonics ASPIC enhanced with MORPHIC's MEMS technology. But we will also construct a large, versatile programmable PIC in which we can define these 


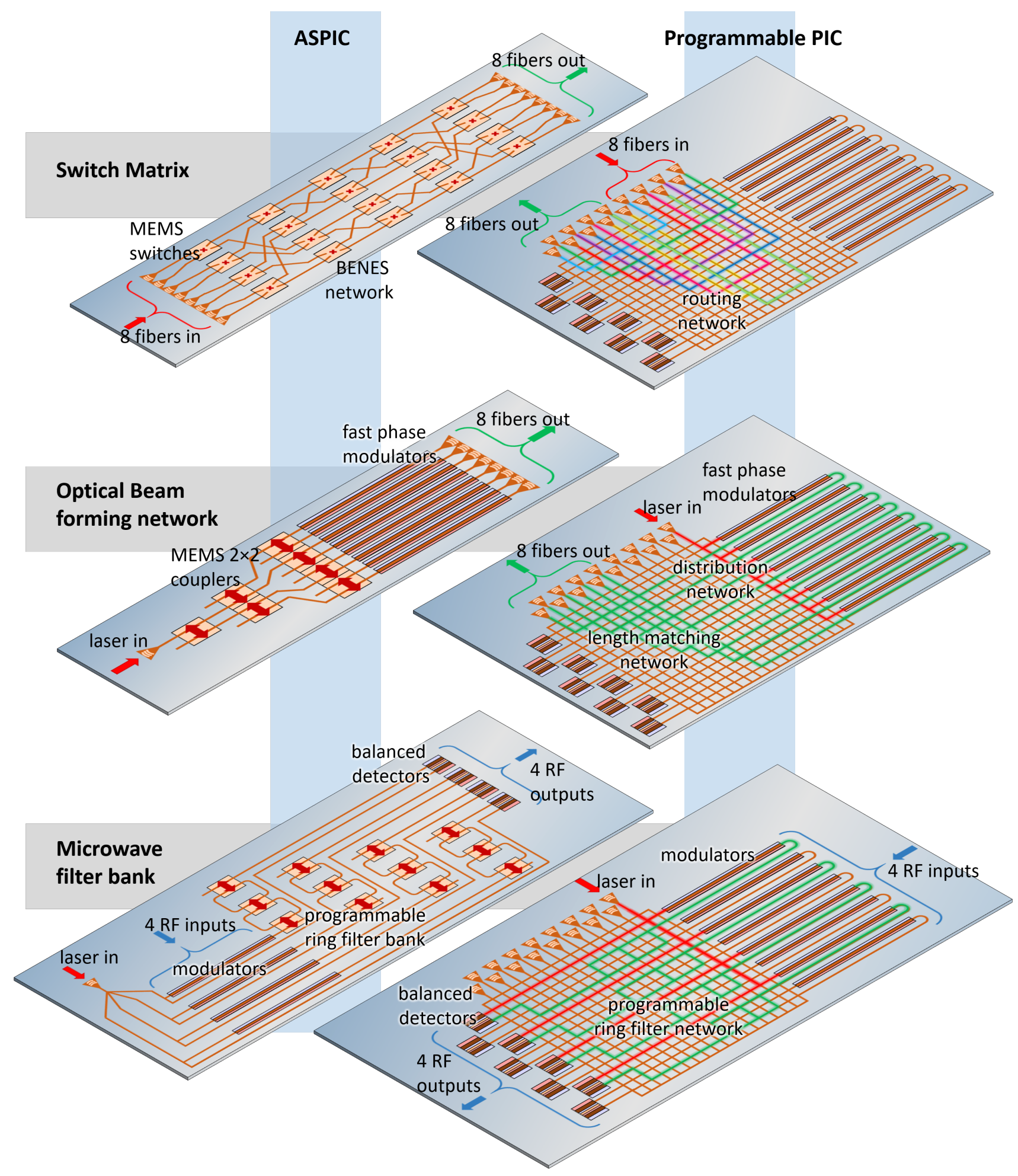

Figure 14. Demonstrator circuits in MORPHIC. We are building demonstrator circuits for three distinct applications: Switch matrices, optical beam forming, and microwave photonics. For each of these applications, we design a dedicated ASPIC using the new MEMS functionality. But we also implement a large generic programmable PIC that can be configured in software to perform these different functions. 
individual demonstrators through software. This will provide a clear comparison of the performance of both circuit architectures and the performance penalties we can expect from programmable PICs.

\section{SUMMARY}

In this paper we gave an overview of the ambitions of the MORPHIC project and the current state of research. By combining MEMS with an existing silicon photonics platform, we aim to overcome a significant bottleneck of silicon photonics technology: the availability of low-power compact electro-optic phase shifters and tunable couplers. This technology is an essential precondition for implementing large-scale programmable photonic circuits, as these consist of waveguide meshes interconnected with exactly those phase shifters an tunable couplers. The choice in MORPHIC to extend the mature iSiPP50G silicon photonics platform allows us to reuse all the developments in this platform, including high-speed modulators and photodetectors.

The integrated MEMS devices, in combination with the large number of circuit elements needed for a programmable circuit, introduces quite some packaging and assembly challenges, which we address with an interposer-based packaging strategy that can handle large numbers of electrical, RF and optical ports. This allows us to interface with the analog and digital driver electronics, and the software layers we are developing to allow the users to program these new programmable PICs.

To demonstrate the versaility of both the MEMS technology and the programmable PIC architectures, we are building demonstrators targeted at different application domains. By implementing a switch matrix, an optical beamformer and a microwave processor in both a custom ASPIC circuit as well as a programming scheme in a generic programmable PIC, we can compare the relative performance of the two photonic circuit concepts.

\section{ACKNOWLEDGMENTS}

This work was funded by the European Union's Horizon2020 program under grant No. 780283 (MORPHIC).

\section{REFERENCES}

[1] Chen, X., Milosevic, M. M., Stankovic, S., Reynolds, S., Bucio, T. D., Li, K., Thomson, D. J., Gardes, F., and Reed, G. T., "The Emergence of Silicon Photonics as a Flexible Technology Platform," Proceedings of the IEEE 106(12), 2101-2116 (2018).

[2] Smit, M., Williams, K., and van der Tol, J., "Past , present , and future of InP-based photonic integration," APL Photonics 050901(May) (2019).

[3] Bogaerts, W. and Chrostowski, L., "Silicon Photonics Circuit Design: Methods, Tools and Challenges," Laser and Photonics Reviews 1700237, 1-29 (2018).

[4] Pérez, D., Gasulla, I., and Capmany, J., "Programmable multifunctional integrated nanophotonics - Review Article," De Gruyter - Nanophotonics 2018 7(8), 1351-1371 (2018).

[5] Reck, M., Zeilinger, A., Bernstein, H. J., and Bertani, P., "Experimental realization of any discrete unitary operator," Phys. Rev. Lett. 73, 58-61 (7 1994).

[6] Miller, D. A. B., "Self-aligning universal beam coupler.," Optics express 21(5), 6360-70 (2013).

[7] Harris, N. C., Carolan, J., Bunandar, D., Prabhu, M., Hochberg, M., Baehr-Jones, T., Fanto, M. L., Smith, A. M., Tison, C. C., Alsing, P. M., and Englund, D., "Linear programmable nanophotonic processors," Optica 5(12), 1623 (2018).

[8] Shen, Y., Harris, N. C., Skirlo, S., Prabhu, M., Baehr-jones, T., Hochberg, M., Sun, X., Zhao, S., Larochelle, H., Englund, D., and Solja, M., "Deep learning with coherent nanophotonic circuits," 11(June) (2017).

[9] Wang, J., Sciarrino, F., Laing, A., and Thompson, M. G., "Integrated photonic quantum technologies," Nature Photonics (2019).

[10] Carolan, J., Harrold, C., Sparrow, C., Martin-Lopez, E., Russell, N. J., Silverstone, J. W., Shadbolt, P. J., Matsuda, N., Oguma, M., Itoh, M., Marshall, G. D., Thompson, M. G., Matthews, J. C. F., Hashimoto, T., O'Brien, J. L., and Laing, A., "Universal linear optics," Science 349(6249), 711-716 (2015).

[11] Clements, W. R., Humphreys, P. C., Metcalf, B. J., Kolthammer, W. S., and Walmsley, I. A., "An Optimal Design for Universal Multiport Interferometers," (2), 1-8 (2016). 
[12] Harris, N. C., Bunandar, D., Pant, M., Steinbrecher, G. R., Mower, J., Prabhu, M., Baehr-Jones, T., Hochberg, M., and Englund, D., "Large-scale quantum photonic circuits in silicon," Nanophotonics 5(3), 456-468 (2016).

[13] Marpaung, D., Yao, J., and Capmany, J., "Integrated microwave photonics," Nature Photonics 13(2), 80-90 (2019).

[14] Pérez, D., Gasulla, I., and Capmany, J., "Toward Programmable Microwave Photonics Processors," Journal of Lightwave Technology 36(2), 519-532 (2018).

[15] Zhuang, L., Roeloffzen, C. G. H., Hoekman, M., Boller, K., and Lowery, A. J., "Programmable photonic signal processor chip for radiofrequency applications," 2(10), 1-6 (2015).

[16] Reed, G. T., Mashanovich, G., Gardes, F. Y., and Thomson, D. J., "Silicon optical modulators," Nature Photonics 4(8), 518-526 (2010).

[17] Verbist, J., Verplaetse, M., Srinivasan, S. A., Van Kerrebrouck, J., De Heyn, P., Absil, P., De Keulenaer, T., Pierco, R., Vyncke, A., Torfs, G., Yin, X., Roelkens, G., Van Campenhout, J., and Bauwelinck, J., "RealTime $100 \mathrm{~Gb} / \mathrm{s}$ NRZ and EDB Transmission with a GeSi Electroabsorption Modulator for Short-Reach Optical Interconnects," Journal of Lightwave Technology 36(1), 90-96 (2018).

[18] Pérez, D., Gasulla, I., and Capmany, J., "Field-programmable photonic arrays," Optics Express 26(21), 27265 (2018).

[19] Masood, A., Pantouvaki, M., Goossens, D., Lepage, G., Verheyen, P., Van Thourhout, D., Absil, P., and Bogaerts, W., "CMOS-compatible Tungsten heaters for silicon photonic waveguides," in [IEEE International Conference on Group IV Photonics GFP], 234-236 (2012).

[20] Hui, Y., Pantouvaki, M., Van Campenhout, J., Korn, D., Komorowska, K., Dumon, P., Li, Y., Verheyen, P., Absil, P., Alloatti, L., Hillerkuss, D., Leuthold, J., Baets, R., and Bogaerts, W., "Performance tradeoff between lateral and interdigitated doping patterns for high speed carrier-depletion based silicon modulators," Opt. Express 20, 12926-12938 (6 2012).

[21] Xing, Y., Ako, T., Member, S., George, J. P., Member, S., Korn, D., Yu, H., Verheyen, P., Pantouvaki, M., Lepage, G., Absil, P., Ruocco, A., Koos, C., Leuthold, J., Neyts, K., and Beeckman, J., "Digitally Controlled Phase Shifter Using an SOI Slot Waveguide With Liquid Crystal Infiltration," Photon. Technol. Lett. 27, 1269-1272 (6 2015).

[22] Abel, S., Eltes, F., Ortmann, J. E., Messner, A., Castera, P., Wagner, T., Urbonas, D., Rosa, A., Gutierrez, A. M., Tulli, D., Ma, P., Baeuerle, B., Josten, A., Heni, W., Caimi, D., Czornomaz, L., Demkov, A. A., Leuthold, J., Sanchis, P., and Fompeyrine, J., "Large Pockels effect in micro- and nanostructured barium titanate integrated on silicon," Nature Materials 18(1), 42-47 (2019).

[23] Errando-Herranz, C., Takabayashi, A. Y., Edinger, P., Sattari, H., Gylfason, K. B., and Quack, N., "MEMS for Photonic Integrated Circuits," IEEE Journal of Selected Topics in Quantum Electronics 26(2), 1-1 (2019).

[24] Wuttig, M., Bhaskaran, H., and Taubner, T., "Phase-change materials for non-volatile photonic applications," Nature Photonics 11(8), 465-476 (2017).

[25] Seok, T. J., Quack, N., Han, S., Muller, R. S., and Wu, M. C., "Highly Scalable Digital Silicon Photonic MEMS Switches," Journal of Lightwave Technology 34, 365-371 (1 2016).

[26] Morichetti, F., Grillanda, S., Carminati, M., Ferrari, G., Sampietro, M., Strain, M. J., Sorel, M., and Melloni, A., "Non-invasive on-chip light observation by contactless waveguide conductivity monitoring," IEEE Journal on Selected Topics in Quantum Electronics 20(4), 292-301 (2014).

[27] Van Acoleyen, K., Roels, J., Mechet, P., Claes, T., Van Thourhout, D., and Baets, R., "Ultracompact phase modulator based on a cascade of NEMS-operated slot waveguides fabricated in silicon-on-insulator," IEEE Photonics Journal 4, 779-788 (6 2012).

[28] Liu, T., Pagliano, F., Van Veldhoven, R., Pogoretskii, V., Jiao, Y., and Fiore, A., "InP MEMS Mach-Zehnder interferometer optical switch on silicon," European Conference on Integrated Optics (ECIO) (2019).

[29] Errando-Herranz, C., Niklaus, F., Stemme, G., and Gylfason, K. B., "Low-power microelectromechanically tunable silicon photonic ring resonator add-drop filter," Optics Letters 40(15), 3556 (2015).

[30] Chu, H. M. and Hane, K., "A wide-tuning silicon ring-resonator composed of coupled freestanding waveguides," IEEE Photonics Technology Letters 26(14), 1411-1413 (2014). 
[31] Abe, S. and Hane, K., "Variable-gap silicon photonic waveguide coupler switch with a nanolatch mechanism," IEEE Photonics Technology Letters 25(7), 675-677 (2013).

[32] Seok, T. J., Kwon, K., Henriksson, J., Luo, J., and Wu, M. C., "Wafer-scale silicon photonic switches beyond die size limit," Optica 6(4), 490 (2019).

[33] Bulgan, E., Kanamori, Y., and Hane, K., "Submicron silicon waveguide optical switch driven by microelectromechanical actuator," Applied Physics Letters 92(10), 1-4 (2008).

[34] Pantouvaki, M., Srinivasan, S. A., Ban, Y., De Heyn, P., Verheyen, P., Lepage, G., Chen, H., De Coster, J., Golshani, N., Balakrishnan, S., Absil, P., and Van Campenhout, J., "Active Components for $50 \mathrm{~Gb} / \mathrm{s}$ NRZ-OOK Optical Interconnects in a Silicon Photonics Platform," Journal of Lightwave Technology 35(4), 631-638 (2017).

[35] Sattari, H., Takabayashi, A., Zhang, Y., and Quack, N., "silicon Photonic Broadband Suspended Directional Coupler," in [2019 International Conference on Optical MEMS and Nanophotonics], 214-215 (2019).

[36] Quack, N., Member, S., Sattari, H., Takabayashi, A. Y., Zhang, Y., Bogaerts, W., Member, S., Edinger, P., Errando-herranz, C., and Gylfason, K. B., "MEMS-enabled Silicon Photonic Integrated Devices and Circuits," IEEE Journal of Quantum Electronics PP, 1 (2019).

[37] Edinger, P., Errando-Herranz, C., and Gylfason, K. B., "Low-Loss MEMS Phase Shifter for Large Scale Reconfigurable Silicon Photonics," Proceedings of the IEEE International Conference on Micro Electro Mechanical Systems (MEMS) 2019-Janua(January), 919-921 (2019).

[38] Unamuno, A. and Uttamchandani, D., "MEMS variable optical attenuator with vernier latching mechanism," IEEE Photonics Technology Letters 18(1), 88-90 (2006).

[39] Wang, X., Bleiker, S. J., Edinger, P., Errando-Herranz, C., Roxhed, N., Stemme, G., Gylfason, K. B., and Niklaus, F., "Wafer-level vacuum sealing by transfer bonding of silicon caps for small footprint and ultra-thin MEMS packages," Journal of Microelectromechanical Systems 28(3), 460-471 (2019).

[40] Ikeda, T. and Hane, K., "A microelectromechanically tunable microring resonator composed of freestanding silicon photonic waveguide couplers," Applied Physics Letters 102(22) (2013).

[41] Ikeda, T. and Hane, K., "silicon photonic waveguide couplers A microelectromechanically tunable microring resonator composed of freestanding silicon photonic waveguide couplers," 221113(2013) (2017).

[42] Ribeiro, A., Ruocco, A., Vanacker, L., and Bogaerts, W., "Demonstration of a 4 X 4-port universal linear circuit," Optica 3(12), 1348 (2016).

[43] Pérez, D., Gasulla, I., Capmany, J., and Soref, R. A., "Reconfigurable lattice mesh designs for programmable photonic processors," Optics Express 24(11), 12093 (2016).

[44] Capmany, J., Gasulla, I., and Pérez, D., "Microwave photonics: The programmable processor," Nature Photonics 10(1), 6-8 (2016).

[45] Zand, I. and Bogaerts, W., "No Title," Photonics Research 8 (2020).

[46] Pérez, D. and Capmany, J., "Scalable analysis for arbitrary photonic integrated waveguide meshes," Optica 6(1), 19 (2019).

[47] Hwang, H. Y., Lee, J. S., Seok, T. J., Forencich, A., Grant, H. R., Knutson, D., Quack, N., Han, S., Muller, R. S., Papen, G. C., Wu, M. C., and O'Brien, P., "Flip Chip Packaging of Digital Silicon Photonics MEMS Switch for Cloud Computing and Data Centre," IEEE Photonics Journal 9(3), 1-10 (2017).

[48] Lambrecht, J., Ramon, H., Moeneclaey, B., Verbist, J., Verplaetse, M., Vanhoecke, M., Ossieur, P., Heyn, P. D., Campenhout, J. V., Bauwelinck, J., and Yin, X., "90-Gb / s NRZ Optical Receiver in Silicon Using a Fully Differential Transimpedance Amplifier," Journal of Lightwave Technology 37(9), 1964-1973 (2019).

[49] Pavarelli, N., Lee, J. S., Rensing, M., Scarcella, C., Zhou, S., Ossieur, P., and Obrien, P. A., "Optical and electronic packaging processes for silicon photonic systems," Journal of Lightwave Technology 33(5), 991-997 (2015).

[50] Zand, I. and Bogaerts, W., "MP6 - Discretization Effects of Digital Control of Thermally Tunable 2x2 MZI Couplers," 2019 IEEE Photonics Society Summer Topical Meeting Series (SUM) , 1-2 (2019).

[51] Chen, X. and Bogaerts, W., "ME2.2 - A Graph-based Design and Programming Strategy for Reconfigurable Photonic Circuits," 2019 IEEE Photonics Society Summer Topical Meeting Series (SUM) , 1-2 (2019).

[52] Lopez, A., Perez, D., Prometheus, D., and Capmany, J., "Auto-routing algorithm for field-programmable photonic gate arrays," 28(1), 737-752 (2020). 